ISSN 1997-5902

\title{
Effet de l'urée et du NPK 15-15-15 perlés et super granulés sur la productivité des variétés de riz IR841 et NERICA-L14 en zone de bas-fond au Sud-Bénin
}

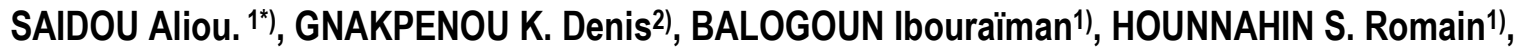 \\ KINDOMIHOU M. Valentin3)
}

1) Unité de Recherche sur la Gestion Intégrée du Sol et des Cultures, Laboratoire des Sciences du Sol, Département de Production Végétale, Faculté des Sciences Agronomiques, Université d'Abomey-Calavi, BENIN ; 03 BP 2819 Jéricho Cotonou, BENIN

2) IFDC NWAFD 04 BP 673 Cotonou, BENIN

3) Unité de Recherche sur l'Ecophysiologie et Amélioration des Plantes Fourragères Tropicales, Laboratoire d'Ecologie Appliquée, Département d'Aménagement et de Gestion de l'Environnement, Faculté des Sciences Agronomiques, Université d'Abomey-Calavi, 03 BP 1974 Jéricho Cotonou, BENIN

*Adresse de l'auteur correspondant: Saïdou Aliou, Tél. (+229) 97494480; Fax. (+229) 21360122 ; Email : saidoualiou@gmail.com

Original submitted in on $8^{\text {th }}$ May 2014. Published online at www.m.elewa.org on $31^{\text {st }}$ May 2014. http://dx.doi.org/10.4314/jab.v77i1.13

\section{RESUME}

Objectifs : La présente étude réalisée sur les sites rizicoles de Koussin et de Lélé au Sud Bénin, examine l'efficacité de l'application de l'urée $(46 \% \mathrm{~N})$ et des engrais NPK 15-15-15 perlés et granulés pour la production du riz de bas-fond. De façon spécifique, l'étude vise à évaluer l'effet des différentes formes d'application de l'urée et des NPK 15-15-15 (granulée et perlée) sur les paramètres de croissance et de rendement de deux variétés de riz (IR841 et NERICA-L14) de bas-fond populaires dans les bas-fonds rizicoles du Bénin.

Méthodologie et résultats : Le dispositif expérimental est un split-plot comportant deux variétés de riz (IR 841 et NERICA-L14), quatre traitements (urée + NPK perlés; urée perlée + NPK super granulé ; urée super granulée + NPK perlé et urée super granulée + NPK super granulé) en quatre répétitions. Les paramètres de croissance et de rendement du riz induits ont été évalués. Les combinaisons (urée super granulée + NPK super granulé) et (urée granulée + NPK perlé) ont induit des hauteurs de plants, un nombre de talles et de grains par panicules significativement $(P<0,0001)$ plus élevés comparativement aux autres traitements quelle que soit la variété de riz. De plus, les rendements moyens de paddy sont significativement $(P<0,0001)$ plus élevés pour les combinaisons urée super granulée + NPK super granulé (riz paddy : $5,52 \pm 0,26$ t/ha ; paille : 12,19 $\pm 0,54$ t/ha) et les combinaisons urée super granulée + NPK perlé (riz paddy: 4,82 $\pm 0,22$ tha ; paille : 10,79 $\pm 0,61$ t/ha). En général, pour les mêmes combinaisons d'engrais, les rendements de paddy et paille les plus élevés ont été produits par la variété IR841.

Conclusion et application: Les engrais super granulés en riziculture de bas-fond sont recommandables pour une meilleure production des variétés améliorées de riz car ils augmentent substantiellement les rendements. Afin d'améliorer l'efficience des engrais, surtout les sources d'azote, l'étude suggère 

des variétés de riz IR841 et NERICA-L14 au Sud-Bénin

l'utilisation de l'urée super granulée + NPK super granulé à défaut l'urée super granulée + NPK perlé pour une amélioration des paramètres de croissance et de rendement de la variété de riz IR841.

Mots clés : Bas-fond, engrais super granulés, fertilité des sols, riziculture irriguée, Bénin.

\section{ABSTRACT}

Effect of pearl and super granule urea and NPK 15-15-15 on the productivity of IR841 and NERICAL14 rice varieties in the inland valley of southern Benin

Objectives: The study was carried out on the site of Koussin and Léle in the southern Benin, exploring the efficiency of the supply of the pearl and super granule urea (46\%) and NPK 15-15-15 for rice production in the inland valley. Specifically, the study aims to assess the effect of the different forms of urea and NPK 1515-15 on the growth and yield parameters of two inland rice varieties (IR841 and NERICA-L14) widely adopted in cultivated lowland of Benin.

Methodology and results: The experimental design is a split-plot with two rice varieties, four forms of fertilizer (pearl urea + NPK; pearl urea + super granule NPK; super granule urea + pearl NPK and super granule urea + super granule NPK) in four replications. The growth and yield parameters were assessed. Super granule urea + super granule NPK and super granule urea + pearl NPK have significantly $(P<$ 0.0001) induced higher plant height, numbers of tiller and rice grain per panicle compared with the other treatments whatever the rice variety. However, mean yields of paddy rice and straw were significantly higher $(P<0,0001)$ for super granule urea + super granule NPK (paddy rice yield : $5,52 \pm 0,26$ t/ha ; straw yield : $12,19 \pm 0,54$ t/ha) and super granule urea + pearl NPK (paddy rice yield: 4,82 $\pm 0,22$ t/ha ; straw yield : $10,79 \pm 0,61 \mathrm{t} / \mathrm{ha}$ ). In general with these treatments, the highest yields were recorded with IR 841 rice variety.

Conclusion and application: The super granule fertilizers are recommended for improving rice production in the inland valley as they improve substantially the yields. In order to improve the efficiency of these fertilizers especially the sources of nitrogen, the study suggests to supply urea super granule + NPK super granule or urea super granule + pearl NPK to improve growth and yields parameter of IR841 rice variety.

Keywords: inland valley, super granule fertilizer, soil fertility, irrigated rice production, Benin.

\section{INTRODUCTION}

Le riz est la première céréale mondiale en terme de consommation alimentaire humaine. Jadis aliment de luxe et consommé en périodes de fêtes, il constitue de nos jours, la base du régime alimentaire dans les pays les moins avancés (FAO, 2004). II constitue avec le blé, les céréales les plus consommées dans le monde (Abiassi, 2006 ; FAOSTAT, 2012). En effet, le riz assure $27 \%$ des disponibilités alimentaires énergétiques et $20 \%$ des apports protéiques dans le monde (FAO, 2004). II fait partie intégrante des systèmes de production agricole en Afrique. Son importance ne cesse de s'accroître, tant au plan alimentaire qu'économique (Totin et al., 2012). II joue un rôle très important dans la contribution à la sécurité alimentaire, importante source de revenus aux producteurs et par conséquent à la réduction de la pauvreté dans certains pays Africains (Fagade,
2000 ; Totin et al., 2013). L'augmentation de la production rizicole mondiale pour approvisionner la population du globe à l'horizon 2020, requiert l'augmentation des rendements en lieu et place de l'augmentation des surfaces qui n'est plus une pratique viable à cause de la forte croissance démographique (Fagade, 2000). Pour cela, l'accent doit être mis sur les facteurs de production (semences améliorées, engrais spécifiques et pesticides). Dans la majorité des pays producteurs de riz, le rapport entre le prix sur le marché international des engrais et celui du riz est élevé (Roger 1993). Cette situation explique l'intérêt de plus en plus croissant que les producteurs accordent aux ressources locales capables de fournir de l'azote bon marché. II est évident que, l'augmentation de la production rizicole ne pourra se faire sans une augmentation globale de 
l'utilisation des engrais de synthèse (Koné et al., 2011). Mais des efforts doivent être consentis afin d'améliorer l'efficience de l'utilisation des nutriments apportés par ces engrais en particulier l'azote. Au Bénin, cette céréale joue un rôle important pour la sécurité alimentaire et dans la réduction de la pauvreté (ADRAO, 2007 ; Totin et al., 2012). En effet, sa consommation par tête d'habitant et par an varie de 6 à 20 kilogrammes en zones rurales contre 10 à 30 kilogrammes en zones urbaines (Adégbola et Sodjinou, 2003). De plus, la production pour la campagne 2009-2010 était de 150.604 tonnes, soit une augmentation de $174 \%$ par rapport à la campagne 2001-2002 au cours de laquelle la production était de 54.901 tonnes (MAEP, 2010). Malgré ces potentialités, la production rizicole au Bénin est caractérisée par un faible niveau de productivité à cause du faible niveau d'utilisation des intrants d'une part, et de l'indisponibilité d'intrants spécifiques en particulier les engrais d'autre part (Adégbola et Sodjinou, 2003 ; Saïdou et Kossou, 2009). Au Bénin, le système de culture à base de riz est essentiellement de bas-fonds associé à une faible riziculture de plateau (Saïdou et Kossou, 2009; Totin et al., 2012) avec des caractéristiques différentes (Ponnamperuma, 1972) avec pour conséquence des types et modes de fertilisations spécifiques. A cet effet, il est impérieux de mettre à la disposition des producteurs des engrais spécifiques adaptés à cet écosystème afin de réduire les pertes de nutriments surtout l'azote par volatilisation et lessivage de l'ammoniac d'une part, et d'accroitre l'efficience de l'utilisation des engrais d'autre part. C'est dans cette perspective que le Centre International pour le Développement des Engrais (IFDC) a entamé un processus d'apprentissage avec les riziculteurs dans les zones inondables du Bénin sur l'utilisation des engrais super granulés. Ce type d'engrais a été

\section{MATERIEL ET METHODES}

Milieu d'étude : L'expérimentation a été conduite dans la commune de Covè, département du Zou, qui est une zone ciblée par les programmes de recherche de l'IFDC-Bénin. Cette commune est limitée à l'Est par la commune de Zagnanado, à l'Ouest par le fleuve Zou expérimenté puis introduit en Asie avec succès pour l'intensification de la production rizicole (Hussain et al., 2010). Cette forme d'engrais répond à la baisse du rendement des cultures au point où l'accès aux engrais spécifiques en riziculture de bas-fonds serait l'une des contraintes majeures à cette culture (IFDC, 2012 ; Totin et al., 2012). L'introduction des engrais super granulés s'inscrit bien dans la problématique de l'intensification de la production agricole dans les systèmes de production qui préoccupe l'IFDC. En effet, depuis 2010, I'IFDC accompagne les producteurs des périmètres rizicoles de Covè (Sud Bénin) dans le processus de fabrication de ce type d'engrais spécifique sur place à partir des engrais conventionnels. Le super granule d'urée est l'une des formes d'engrais azotés largement adoptées par les producteurs asiatiques (Hussain et al., 2010). II peut être appliqué dans la zone racinaire du riz de 8 à $10 \mathrm{~cm}$ de profondeur du sol. Cette pratique réduit de $30 \%$ les pertes d'azote et augmente le taux de recouvrement de l'engrais tout en limitant la pollution des sols et des eaux et par conséquent, contribue à une augmentation du rendement de riz (Pasandaran et al., 1999 ; IFDC, 2003 ; Mohanty et al., 1999; Chowdhury et al., 2009). La présente étude s'inscrit dans la perspective de mettre au point des formes d'engrais spécifique adaptées en riziculture de bas-fond au Bénin. De façon spécifique, l'étude vise à : 1) évaluer l'effet des différentes formes d'application de l'urée et du NPK 15-15-15 (granulée et perlée) sur les paramètres de croissance de deux variétés de riz (IR841 et NERICA-L14) de bas-fond largement cultivées ; 2) déterminer l'effet des différentes formes d'application de l'urée et de NPK (granulée et perlée) sur les rendements en riz paddy et en paille de deux variétés de riz (IR 841 et NERICAL14) de bas-fond.

(qui la sépare des communes de Djidja et de Za-kpota), au Sud par la commune de Zogbodomey et au Nord par la commune de Dassa-Zoumè (Figure 1). Le périmètre rizicole regroupe les sites de Koussin et celui de Lélé distants l'un de l'autre de $4 \mathrm{~km}$. Les parcelles 
expérimentales de Koussin sont situées à $7^{\circ} 14$ au Nord et $02^{\circ} 17$ à l'Est.

Nord et $02^{\circ} 17$ à l'Est; celles de Lélé sont à $7^{\circ} 16$ au

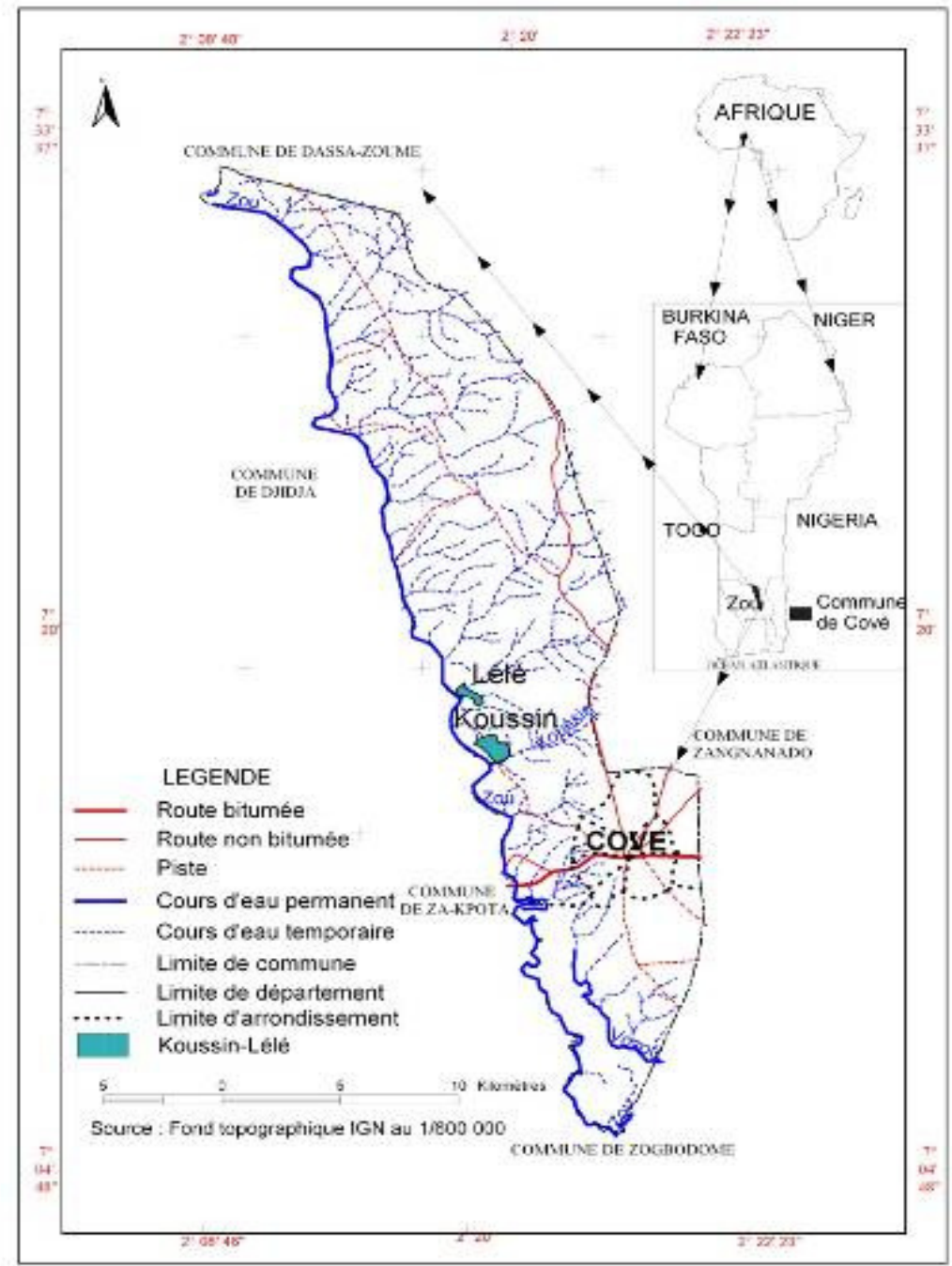

Figure 1: Carte de la commune de Covè au Bénin avec la localisation des périmètres rizicoles de Koussin et de Lélé.

La zone d'étude jouit d'un climat de transition entre le climat subéquatorial maritime et le climat soudanoguinéen du nord Bénin. Les sols des périmètres rizicoles de Koussin et de Lélé sont hydromorphes minéraux à gley de profondeur (CPCS, 1967) à drainage modéré, brun moyennement humifère limonoargileux (PAGFS/MAEP, 2010). Ce sont des sols de fertilité physique moyenne à élevée. Leurs caractéristiques physico-chimiques se présentent comme suit : $\mathrm{pH}$ (eau) 6,6 ; matière organique $2,36 \%$; azote-total $0,114 \%$; $\mathrm{K}^{+}$échangeable $0,57 \mathrm{cmol} / \mathrm{kg}$; CEC $17,65 \mathrm{cmol} / \mathrm{kg}$ et $P$ (Bray 1) $28 \mathrm{ppm}$. En période sèche, ils présentent de larges fentes jusqu'à $40 \mathrm{~cm}$ en profondeur. Ces fentes larges de plus ou moins 2 à 4 $\mathrm{cm}$, se remplissent des matières sèches et d'eau. C'est un sol qui, après le retrait des eaux se montre très propice à la culture du riz. En période de crue, la fertilité des sols se trouve améliorée par l'apport d'importantes quantités de sédiments. Cette richesse se renouvelle de manière récurrente par charriage d'abondants alluvions et autres éléments fertilisants à l'occasion des crues du fleuve Zou.

Matériel végétal et formes d'engrais minéraux : Les variétés de riz IR841 à grains aromatiques et NERICAL14 à grains non aromatiques et de cycle 120 jours ont été expérimentées. Les semences ont été acquises à 
Saidou et al. J. Appl. Biosci. 2014. Effet de l'urée et du NPK perlés et super granulés sur la productivité des variétés de riz IR841 et NERICA-L14 au Sud-Bénin

l'Institut National des Recherches Agricoles du Bénin (INRAB), centre de Bohicon. Les engrais super granulés utilisés ont été fabriqués à partir de l'urée $(46 \% \mathrm{~N})$ et du NPK (15-15-15) perlés. Les supers granulés d'urée et de NPK ont été fabriqués à l'aide d'un briqueteur model BM 450 triphasée de 440 volts importé du Bengladesh par IFDC en 2009. Cet appareil compresse les engrais perlés en comprimés supers granulés (Figure 2). La masse et la forme des engrais supers granulés produits dépendent des mailles de la machine.

Dispositif expérimental: Le dispositif expérimental utilisé au niveau des sites de Koussin et de Lélé est un split plot à quatre répétitions. Par site, les variétés de riz (IR841 et NERICA-L14) sont les facteurs principaux et les différentes formes d'urée et de NPK (granulées et perlées) comme facteurs secondaires. Le Tableau 1 présente la description des traitements d'engrais appliquées. La fertilisation a été faite sous la forme de fumure de couverture. La quantité d'urée super granulée correspondant appliquée aux quatre plants était de 2,7 $\mathrm{g}$ et celle de NPK 15-15-15 était de $4 \mathrm{~g}$ correspondant au différentes doses du Tableau 1. Ces doses ont été déterminées sur la base des recommandations d'engrais pour la culture du riz en zone de bas-fonds (IFDC, 2012).
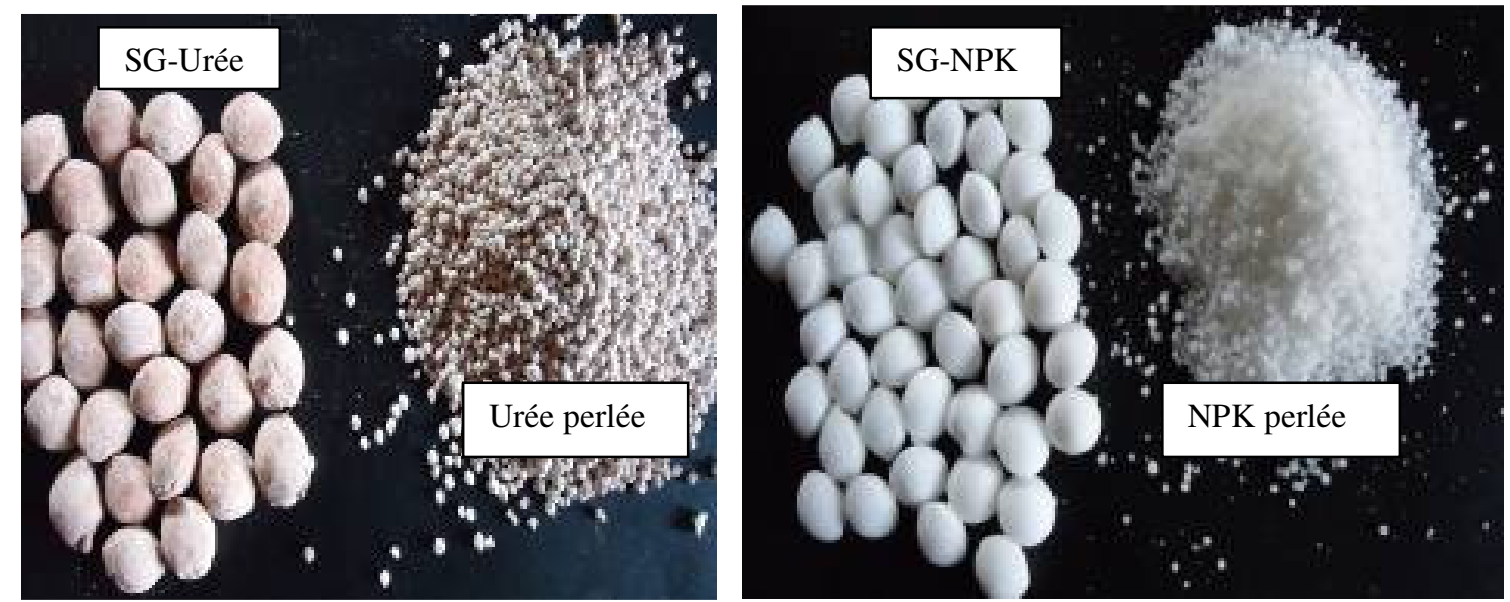

Figure 2 : Urée perlée et super granulée et engrais NPK perlé et super granulé

Tableau 1 : Formes, doses d'engrais (g/parcelle élémentaire) et quantités de nutriments $\mathrm{N}, \mathrm{P}$ et $\mathrm{K}$ appliquées selon les traitements d'engrais et par variété de riz.

\begin{tabular}{l|l|l|l|l|l|l|l}
\hline Traitements & $\begin{array}{l}\text { Urée super } \\
\text { granule }\end{array}$ & $\begin{array}{l}\text { Urée } \\
\text { perlée }\end{array}$ & $\begin{array}{l}\text { NPK super } \\
\text { granule }\end{array}$ & \multicolumn{2}{l|}{ NPK perlé } & \multicolumn{2}{|l|}{$\begin{array}{l}\text { Dose de nutriments } \\
\text { (kg/ha) }\end{array}$} \\
\cline { 5 - 8 } & & & & & N & P & K \\
\hline Urée + NPK perlés & - & 169 & - & 250 & 115,2 & 16,4 & 31,3 \\
Urée perlée + SG-NPK & - & 169 & 250 & - & 115,2 & 16,4 & 31,3 \\
SG-Urée + NPK perlé & 169 & - & - & 250 & 115,2 & 16,4 & 31,3 \\
SG-Urée + SG-NPK & 169 & - & 250 & - & 115,2 & 16,4 & 31,3 \\
\hline
\end{tabular}

NB : SG-NPK = engrais complexe NPK super granulé ; SG-Urée = Urée super granulée ; - = Non applicable

Les engrais perlés ont été appliquées selon la pratique paysanne à la volée après un drainage de l'excès d'eau puis les casiers ont été remplis de nouveau d'eau après deux semaines. Les parcelles élémentaires (casiers) mesurent chacune $5 \mathrm{~m} \times 5 \mathrm{~m}$. Des allées de $0,5 \mathrm{~m}$ de large ont été observées entre les casiers et des allées de $1 \mathrm{~m}$ de large entre deux répétitions.

Conduite de l'essai : Les parcelles expérimentales sur les deux sites ont été labourées au moyen d'un motoculteur les 6 et 10 décembre 2011. Chaque variété de riz a été multipliée en pépinière du 27 novembre au 23 décembre 2011. Le repiquage des plants de riz a été effectué le 24 décembre 2011, soient 28 jours après la mise en pépinière à raison de deux plants par poquet. Les écartements de repiquage étaient de 20 $\mathrm{cm} \times 20 \mathrm{~cm}$ afin de favoriser l'application des engrais super granulés selon les recommandations (Roger, 1993). Le NPK perlé, l'urée super granulée (SG-Urée) 
et le NPK super granulé (SG-NPK) ont été apportés le 3 janvier 2012 soient 10 jours après le repiquage des plants de riz tandis que, la dose d'urée perlée a été fractionnée en deux apports égaux. La première application a été effectuée le 8 janvier 2012 soient 15 jours après le repiquage des plants de riz (début de tallage). La seconde application a été réalisée le 7 février 2012 soient 45 jours après le repiquage (fin de montaison et début d'initiation paniculaire). Les engrais super granulés ont été manuellement déposé au sol

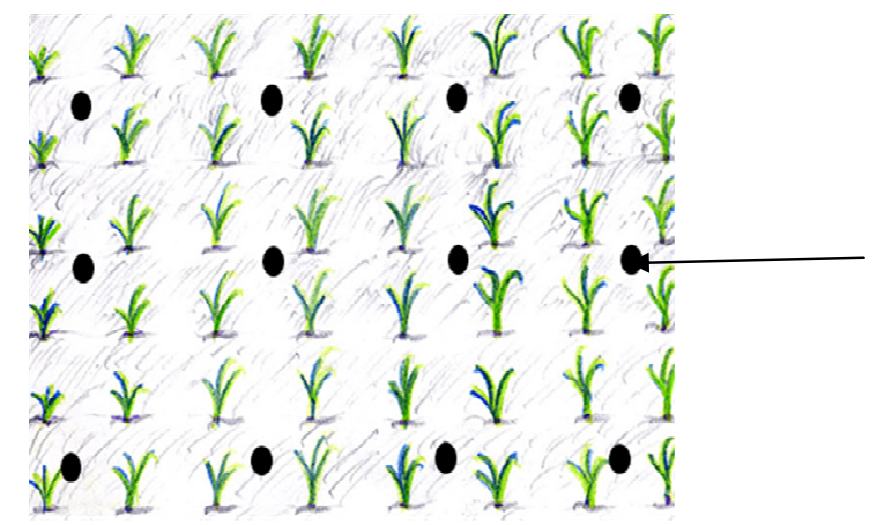

inondé à 7-10 cm de profondeur selon les recommandations de l'IFDC à raison d'un comprimé d'engrais super granulé au centre de quatre poquets de riz (Figure 3). L'entretien des parcelles a consisté à l'arrachage à la main des adventices chaque fois qu'elles apparaissent jusqu'à la maturité physiologique des plants de riz. En suite, une chasse aviaire a été organisée afin de réduire l'effet de l'attaque des oiseaux granivore.

Figure 3 : Schéma montrant le placement des comprimés d'engrais super granulés entre les plants de riz. Un écartement de 0,15 $\mathrm{m} \times 0,15 \mathrm{~m}$ respectivement entre deux plants et entre deux lignes de semis est observé.

Données collectées : Le nombre de talles par poquet a été relevé à la floraison et uniquement sur les tiges florifères, ce qui correspond au nombre de tiges secondaires émergeant du collet de la tige principale. Le comptage s'est effectué au niveau de dix poquets sélectionnés au hasard au niveau de la surface interprétable de chaque unité parcellaire après avoir éliminé les lignes et les plants de bordure. La hauteur des plants (du collet à la dernière ligule) a été mesurée au niveau des dix poquets sélectionnés de façon aléatoire tous les dix jours, à partir du 30 ème jour après repiquage (JAR) jusqu'à la maturité des grains. Le nombre de grains fertiles par panicule a été relevé à la maturité physiologique sur des plants au niveau des dix poquets échantillonnés. Les grains fertiles bien remplis sont relevés après une légère pression sur le paddy. Les grains fertiles sont bien remplis lorsque le grain résiste à la pression des doigts. Le rendement du riz paddy et de la paille à la récolte a été mesuré au niveau de la surface interprétable de chaque unité parcellaire. Les pailles de riz mature ont été coupées, mis en botte puis séchées au soleil au champ afin de réduire le taux d'humidité. Les bottes de paille séchée ont été par la suite battues puis vannées. Les grains paddy résultant du vannage ont été séchés sur l'aire de séchage du périmètre rizicole pendant 72 heures avant d'être pesés pour la détermination du rendement en riz paddy. La paille obtenue après battage a été également pesée afin d'obtenir le rendement paille ceci après un séchage d'une durée de 72 heures sur l'aire de séchage.

Traitements des données: Le logiciel Statistical Analysis System (SAS) version 9.2 a été utilisé pour l'analyse des données. Des analyses de variance (ANOVA) à trois facteurs considérant les sites d'essai, les variétés de riz et les formes d'engrais minéraux appliquées ont été effectuées afin d'apprécier les effets de chaque facteur ainsi que ceux des interactions sur les paramètres mesurés. Les moyennes ont été comparées par le test de Student Newman-Keuls au seuil de $5 \%$ pour la valeur de $a=0,05$. 
RESULTATS

Effet des formes d'engrais et des variétés de riz sur la croissance en hauteur des plants: Les résultats de l'analyse de la variance (Tableau 2) indiquent des différences significatives $(P<0,05)$ à très hautement significatives $(P<0,0001)$ entre les deux sites de production en ce qui concerne les mesures de la hauteur des plants de 30 à 70 JAR.

Tableau 2 : Résultats de l'analyse de la variance (valeur de F) sur la hauteur des plants à différentes périodes de mesure considérant les formes d'engrais appliquées, les variétés et les sites d'expérimentation.

\begin{tabular}{|c|c|c|c|c|c|c|}
\hline \multirow{2}{*}{ Sources de variation } & \multirow{2}{*}{ ddl } & \multicolumn{5}{|c|}{ Valeurs de F } \\
\hline & & 30 JAR & 50 JAR & 70 JAR & 80 JAR & $90 \mathrm{JAR}$ \\
\hline Engrais & 3 & $32,00^{\star * *}$ & $380,25^{\star \star \star}$ & $62,39^{\star \star \star}$ & $153,34^{\star \star \star}$ & $130,41^{\text {***}}$ \\
\hline Variétés & 1 & $0,15 \mathrm{~ns}$ & $3,49 \mathrm{~ns}$ & $15,97^{\star \star *}$ & $0,22 \mathrm{~ns}$ & $3,88 \mathrm{~ns}$ \\
\hline Sites & 1 & $67,43^{\star *}$ & $353,30^{* * *}$ & $22,19^{*}$ & $0,00 \mathrm{~ns}$ & $0,26 \mathrm{~ns}$ \\
\hline Engrais*Variétés & 3 & $0,19 \mathrm{~ns}$ & $0,23 \mathrm{~ns}$ & $0,21 \mathrm{~ns}$ & $0,37 \mathrm{~ns}$ & $0,21 \mathrm{~ns}$ \\
\hline Engrais*Sites & 3 & $2,88^{*}$ & $136,69^{* * *}$ & $0,58 \mathrm{~ns}$ & $11,75^{\star * *}$ & $8,35^{\star * *}$ \\
\hline Variétés*Sites & 1 & $0,40 \mathrm{~ns}$ & $1,71 \mathrm{~ns}$ & $12,73^{*}$ & $1,42 \mathrm{~ns}$ & $8,62^{*}$ \\
\hline Engrais*Variétés*Sites & 3 & $0,50 \mathrm{~ns}$ & $0,30 \mathrm{~ns}$ & $1,16 \mathrm{~ns}$ & $0,40 \mathrm{~ns}$ & $0,21 \mathrm{~ns}$ \\
\hline
\end{tabular}

N.B : JAR= jours après repiquage $;$ ns $=$ non significatif ; ${ }^{*}=P<0,05 ;{ }^{* *}=P<0,01 ;{ }^{* * *}=P<0,0001 ; d d l=$ degré de liberté

Les variétés n'ont eu des effets très significatifs $(P<$ 0,0001 ) sur la hauteur des plants qu'à 70 JAR par contre, les formes d'engrais minéraux appliquées ont très significativement $(P<0,0001)$ affecté la hauteur des plants lors de toutes les périodes de mesure. La Figure 4 présente la croissance en hauteur des plants de riz suivant les formes d'engrais appliquées sur les deux sites d'essais. L'analyse des résultats des graphes révèle que, selon le test de Student NewmanKeuls et à toutes les périodes de mesure, les combinaisons SG-Urée + SG-NPK et SG-Urée + NPK comparativement aux autres combinaisons d'engrais au niveau des deux variétés de riz ont présenté les hauteurs les plus importantes. II ressort également de la figure que, les plus faibles hauteurs sont obtenues avec les combinaisons d'engrais Urée + NPK perlés. Sous ces deux combinaisons d'engrais, au niveau des résultats de la variété IR841 au 50ème JAR sur les deux sites, les hauteurs des plants sont 1,4 à 2 fois significativement plus élevées que celles de la variété NERICAL-14. Toutefois, cette dernière connait remarquablement en 20 jours (c.-à-d. au 70 ème JAR) une croissance en hauteur de $123 \%$ et $141 \%$ respectivement à Lélé et Koussin.

Effet des formes d'engrais et des variétés de riz sur la production de talles et le nombre de grains par panicule mature: Les résultats de l'analyse de la variance du nombre de talles par poquet et du nombre de grains par panicule mature (Tableau 3) considérant les formes d'engrais, les variétés et les sites d'expérimentation montrent que les formes d'engrais ont eu des effets très significatifs $(P<0,0001)$ aussi bien sur le nombre de talles que sur le nombre de grains par panicule. Les sites par contre, n'ont eu des effets significatifs $(P<0,05)$ que sur le nombre de talles. 


\section{Site de Koussin}
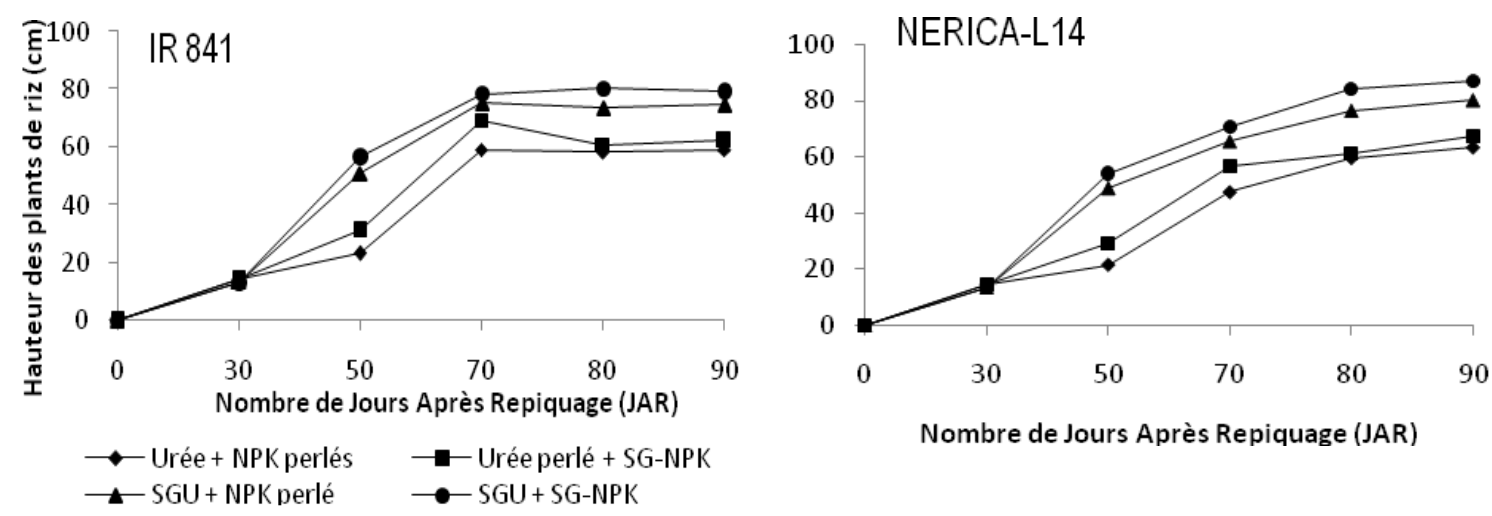

\section{Site de Lélé}
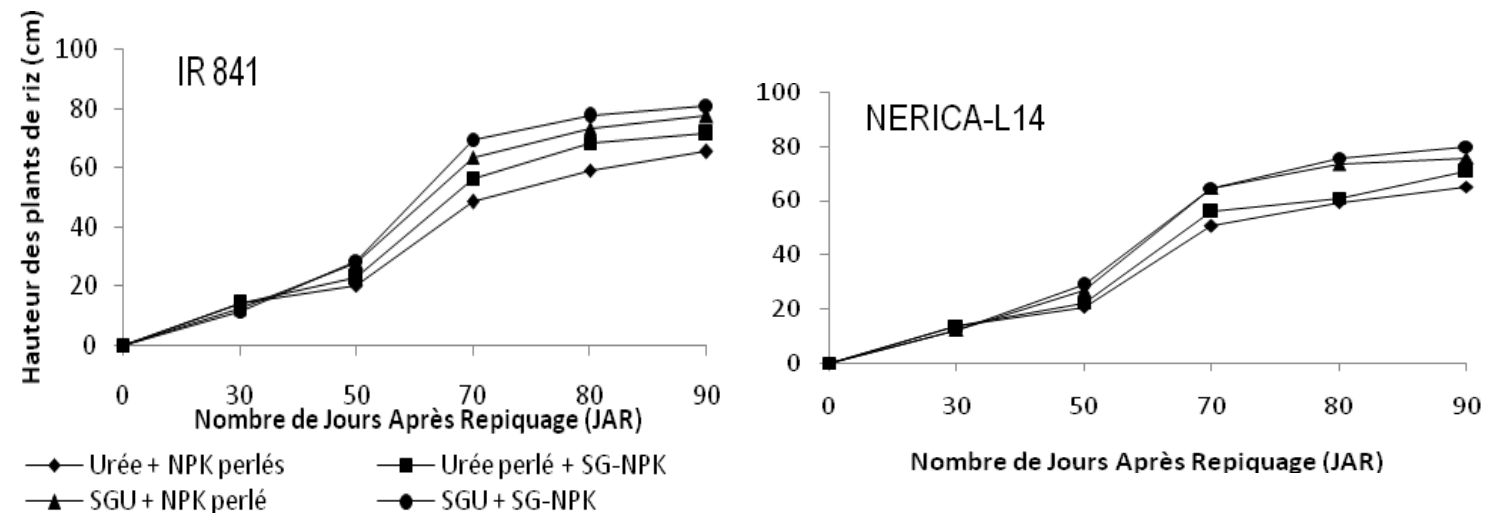

Figure 4: Effet des formes d'engrais minéraux sur la hauteur des plants des variétés de riz IR841 et NERICA-L14 à différentes périodes de mesure sur les sites de Koussin et de Lélé au Bénin

II ressort également de la figure que, les plus faibles hauteurs sont obtenues avec les combinaisons d'engrais Urée + NPK perlés. Sous ces deux combinaisons d'engrais, au niveau des résultats de la variété IR841 au 50ème JAR sur les deux sites, les hauteurs des plants sont 1,4 à 2 fois significativement plus élevées que celles de la variété NERICAL-14. Toutefois, cette dernière connait remarquablement en 20 jours (c.-à-d. au 70ème JAR) une croissance en hauteur de $123 \%$ et $141 \%$ respectivement à Lélé et Koussin.

Effet des formes d'engrais et des variétés de riz sur la production de talles et le nombre de grains par panicule mature: Les résultats de l'analyse de la variance du nombre de talles par poquet et du nombre de grains par panicule mature (Tableau 3) considérant les formes d'engrais, les variétés et les sites d'expérimentation montrent que les formes d'engrais ont eu des effets très significatifs $(P<0,0001)$ aussi bien sur le nombre de talles que sur le nombre de grains par panicule. Les sites par contre, n'ont eu des effets significatifs $(P<0,05)$ que sur le nombre de talles. Les résultats du Tableau 4 indiquent que, sur les deux sites d'expérimentation, le nombre de talles et de grains par panicules matures sont significativement plus élevés $(P<0,05)$ avec les combinaisons comportant l'urée super granulée (SG-Urée + SG-NPK et SG-Urée + NPK perlé) comparativement aux autres combinaisons d'engrais. 
Tableau 3 : Résultats de l'analyse de la variance (valeur de F) sur le nombre de talles par poquet et le nombre de grains de riz par panicule mature considérant les formes d'engrais appliquées, les variétés et les sites d'expérimentation.

\begin{tabular}{lccc}
\hline Source de variation & Ddl & \multicolumn{2}{c}{ Valeurs de F } \\
\cline { 3 - 4 } & & Nombre de talles par poquet & Nombre de grain de riz par panicule \\
\hline Engrais & 3 & $45,84^{* * *}$ & $44,11^{* * *}$ \\
Variétés & 1 & $3,56 \mathrm{~ns}$ & $2,31 \mathrm{~ns}$ \\
Sites & 1 & $13,38^{*}$ & $3,27 \mathrm{~ns}$ \\
Engrais*Variétés & 3 & $0,40 \mathrm{~ns}$ & $0,52 \mathrm{~ns}$ \\
Engrais*${ }^{*}$ Sites & 3 & $0,69 \mathrm{~ns}$ & $1,42 \mathrm{~ns}$ \\
Variétés $^{*}$ Sites & 1 & $7,86 \mathrm{~ns}$ & $2,66 \mathrm{~ns}$ \\
Engrais $^{*}$ Variétés & & $1,80 \mathrm{~ns}$ & $0,40 \mathrm{~ns}$
\end{tabular}

N.B : ns $=$ non significatif ; ${ }^{*}=\mathrm{P}<0,05 ;{ }^{* * *}=\mathrm{P}<0,0001 ; \mathrm{ddl}=$ degré de liberté

En effet, au niveau des deux sites, le nombre total de grain de riz par panicule est significativement 1,4 à 1,5 fois plus élevé au niveau du traitement SG-Urée + SGNPK comparativement à l'utilisation de l'urée + NPK perlés. Toutefois, on note une meilleure performance de la variété IR841 à l'application des engrais super granulés comparativement à la variété NERICA-L14.

Tableau 4 : Effet des variétés et des formes d'application des engrais sur le nombre de talles et le nombre de grains par panicule ( \pm erreurs standards) des plants de riz sur les sites rizicoles de Koussin et Lélé au Bénin.

\begin{tabular}{|c|c|c|c|c|}
\hline Sites & Variétés & $\begin{array}{l}\text { Formes d'applications } \\
\text { des engrais }\end{array}$ & Nombre de talles par poquet & $\begin{array}{l}\text { Nombre de grains } \\
\text { par panicule }\end{array}$ \\
\hline \multirow{7}{*}{ Koussin } & \multirow{3}{*}{ IR 841} & $\begin{array}{l}\text { Urée + NPK perlés } \\
\text { Urée perlé + SG-NPK } \\
\text { SG-Urée + NPK perlés }\end{array}$ & $\begin{array}{l}7,07 \pm 0,40 b \\
9,30 \pm 0,54 b \\
13,25 \pm 0,68 a\end{array}$ & $\begin{array}{l}123,72 \pm 1,58 \mathrm{~b} \\
126,20 \pm 5,36 \mathrm{~b} \\
156,52 \pm 10,70 \mathrm{ab}\end{array}$ \\
\hline & & SG-Urée + SG-NPK & $14,47 \pm 0,64 a$ & $170,32 \pm 15,82 a$ \\
\hline & & Moyenne & $11,02 \pm 0,81 \mathrm{~B}$ & $144,19 \pm 6,72 \mathrm{~A}$ \\
\hline & \multirow{4}{*}{ NERICA-L14 } & $\begin{array}{l}\text { Urée + NPK perlés } \\
\text { Urée perlé + SG-NPK } \\
\text { SG-Urée + NPK perlés }\end{array}$ & $\begin{array}{l}8,75 \pm 0,76 \mathrm{~d} \\
11,60 \pm 0,8 \mathrm{c} \\
15,50 \pm 0,57 \mathrm{~b}\end{array}$ & $\begin{array}{l}124,05 \pm 1,71 b \\
127,25 \pm 3,99 b \\
158,05 \pm 12,07 a b\end{array}$ \\
\hline & & SG-Urée + SG-NPK & $18,60 \pm 0,91 a$ & $170,12 \pm 15,14 a$ \\
\hline & & Moyenne & $13,61 \pm 1,03 \mathrm{~A}$ & $144,87 \pm 6,75 \mathrm{~A}$ \\
\hline & & Moyenne générale & $12,32 \pm 0,68 \mathrm{Y}$ & $144,53 \pm 4,68 X$ \\
\hline \multirow{10}{*}{ Lélé } & \multirow{5}{*}{ IR 841} & Urée + NPK perlés & $11,95 \pm 0,34 d$ & $116,32 \pm 13,00 b$ \\
\hline & & Urée perlé + SG-NPK & $14,05 \pm 0,36 c$ & $126,47 \pm 12,01 b$ \\
\hline & & SG-Urée + NPK perlés & $16,47 \pm 0,78 b$ & $141,27 \pm 5,29 a b$ \\
\hline & & SG-Urée + SG-NPK & $19,95 \pm 0,86 a$ & $169,32 \pm 10,35 a$ \\
\hline & & Moyenne & $15,61 \pm 0,82 \mathrm{~A}$ & $138,35 \pm 7,00 \mathrm{~A}$ \\
\hline & \multirow[t]{5}{*}{ NERICA-L14 } & $\begin{array}{l}\text { Urée + NPK perlés } \\
\text { Urée perlé + SG-NPK }\end{array}$ & $\begin{array}{l}11,17 \pm 0,58 a \\
14,35 \pm 0,66 a\end{array}$ & $\begin{array}{l}100,57 \pm 8,40 \mathrm{c} \\
114,90 \pm 5,08 \mathrm{bc}\end{array}$ \\
\hline & & SG-Urée + NPK perlés & $17,90 \pm 1,01 a$ & $124,72 \pm 3,79 a b$ \\
\hline & & SG-Urée + SG-NPK & $16,97 \pm 5,60 \mathrm{a}$ & $138,25 \pm 5,60 \mathrm{a}$ \\
\hline & & Moyenne & $15,10 \pm 1,09 \mathrm{~A}$ & $119,61 \pm 4,44 \mathrm{~B}$ \\
\hline & & Moyenne générale & $15,35 \pm 0,67 X$ & $128,98 \pm 4,11 X$ \\
\hline
\end{tabular}



des variétés de riz IR841 et NERICA-L14 au Sud-Bénin

Les moyennes suivies des mêmes lettres alphabétiques de même caractère et pour les mêmes caractéristiques ne sont pas significativement différentes $(P>0,05)$ d'après le test de Student Newman-Keuls. SG-Urée $=$ Urée super granulée ; SG-NPK = NPK super granulée.

Globalement, sur le site de Koussin, la variété IR841 présente un nombre de talles significativement 1,3 fois plus élevés que ceux de la variété NERICA-L14. Au niveau du site de Lélé, le nombre de grains par panicule est significativement 1,2 fois plus élevé avec la variété IR841 comparativement à la variété NERICAL14. Les amplitudes de variations entre les deux sites pour le nombre de grain par panicule mature et de talles sont respectivement de $13 \%$ et de $25 \%$.

Tableau 5 : Résultats de l'analyse de la variance (valeur de F) sur les rendements riz paddy et paille considérant les formes d'engrais appliquées, les variétés et les sites d'expérimentation.

\begin{tabular}{|c|c|c|c|}
\hline \multirow[t]{2}{*}{ Source de variation } & \multirow[t]{2}{*}{ ddl } & \multicolumn{2}{|c|}{ Valeur de F } \\
\hline & & Rendement riz paddy & Rendement paille \\
\hline Engrais & 3 & $37,66^{\star \star \star}$ & $64,01^{* * *}$ \\
\hline Variétés & 1 & $0,93 \mathrm{~ns}$ & $0,53 \mathrm{~ns}$ \\
\hline Sites & 1 & $47,55^{\star \star *}$ & $0,62 \mathrm{~ns}$ \\
\hline Engrais*Variétés & 3 & $0,53 \mathrm{~ns}$ & $0,31 \mathrm{~ns}$ \\
\hline Engrais*Sites & 3 & $1,32 \mathrm{~ns}$ & $7,78^{* * *}$ \\
\hline Variétés*Sites & 1 & $0,03 \mathrm{~ns}$ & $24,05^{\star \star *}$ \\
\hline Engrais ${ }^{*}$ Variétés*Sites & 3 & $0,77 \mathrm{~ns}$ & $1,24 \mathrm{~ns}$ \\
\hline
\end{tabular}

N.B : ns $=$ non significatif $;{ }^{* * *}=\mathrm{P}<0,0001 ; \mathrm{ddl}=$ degré de liberté

Effet des formes d'engrais et des variétés de riz sur les rendements grains de riz paddy et paille: Les résultats de l'analyse de la variance (Tableau 5) indiquent des différences très hautement significatives $(P<0,0001)$ entre les formes d'engrais minéraux appliquées en ce qui concerne les rendements grains de riz paddy et paille. Par contre, des différences très hautement significatives $(P<0,0001)$ n'ont été observées entre les deux sites de production uniquement avec le rendement grain de riz paddy. Les résultats ne montrent aucune discrimination significative $(P>0,05)$ entre les deux variétés. Toutefois, des interactions très hautement significatives $(P<0,0001)$ sont observées entre les sites et les variétés d'une part, et entre les sites et les formes d'engrais minéraux appliquées d'autre part. Les résultats du test de Student Newman-Keuls en rapport avec les rendements grains de riz paddy et paille selon les sites, les variétés et les formes d'engrais minéraux appliquées sont présentés dans la Figure 5. L'analyse des résultats de ces graphes confirme les résultats précédents. En effet, quelque soient les variétés et les sites, la combinaison d'engrais SG-Urée + SG-NPK présente les rendements grains de riz paddy et paille significativement $(P<0,0001)$ plus élevés $(6,43 \pm 0,27$ tha et $12,05 \pm 0,98$ t/ha respectivement pour le rendement grain de riz paddy et paille sur le site de Koussin contre $5,16 \pm 0,22$ tha et $12,07 \pm 1,00$ tha sur le site de Lélé avec la variété IR841) comparativement aux autres traitements. Les rendements grain de riz paddy et paille sont moins importants avec la variété NERICA-L14 $(5,77 \pm 0,72$ tha et $14,33 \pm 0,32$ tha respectivement pour le rendement grain de riz paddy et paille sur le site de Koussin contre 4,73 $\pm 0,37$ t/ha et 10,32 $\pm 1,05$ tha sur le site de Lélé). 


\section{Site de Koussin}
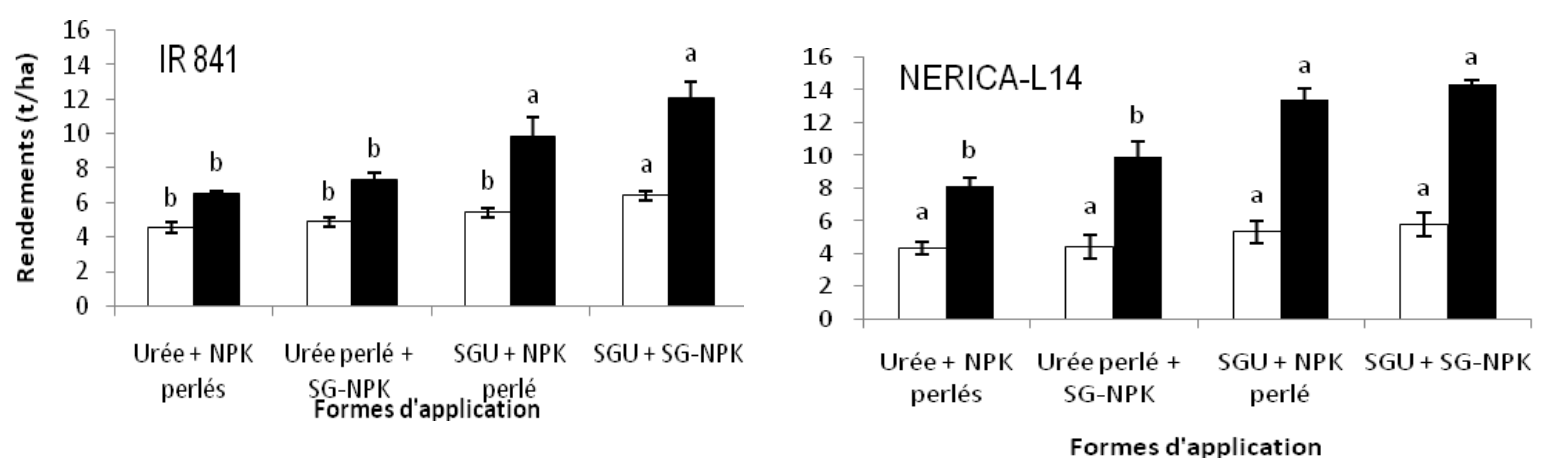

\section{Site de Lélé}
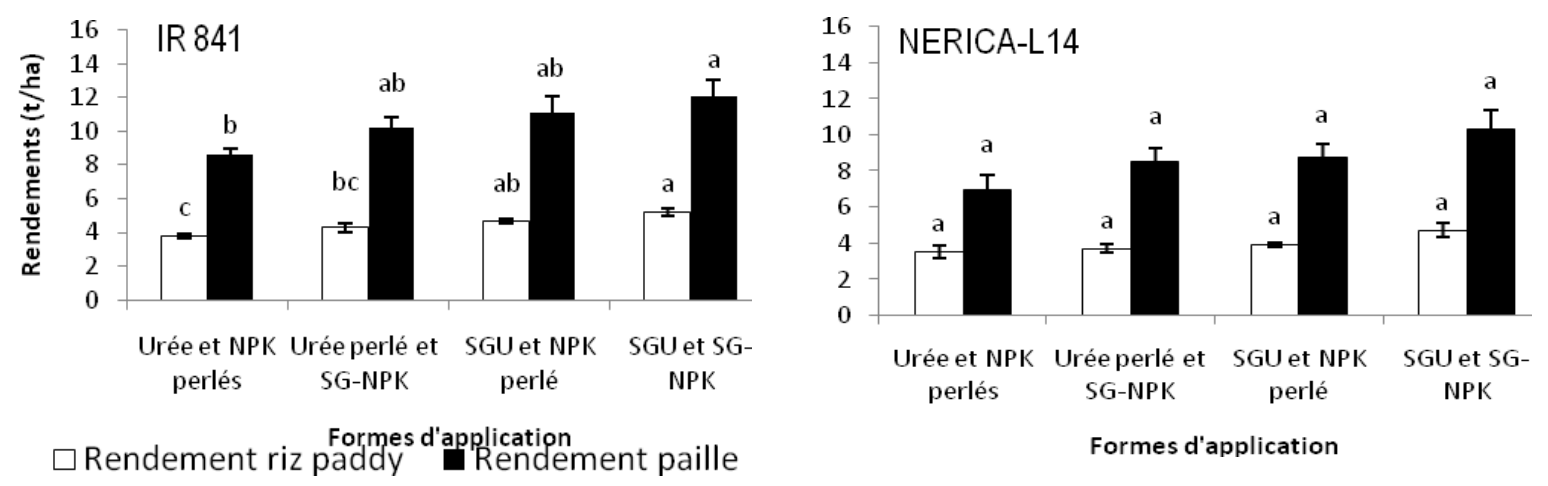

Figure 5 : Effet des formes d'engrais minéraux sur les rendements de riz paddy et paille des variétés IR841 et NERICA-L14 sur les sites de Koussin et de Lélé au Bénin. Les barres d'erreur représentent les erreurs standards. Les barres portant la même lettre alphabétique ne sont pas significativement $(P>0,05)$ différentes selon le test de Student Newman-Keuls.

En général, les plus faibles rendements sont obtenus avec les engrais perlés spécifiquement avec les traitements comportant l'urée perlée. Avec la variété IR841, les rendements grain de riz paddy et paille

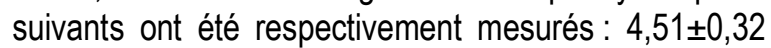
tha et $6,55 \pm 0,07$ tha sur le site de Koussin et $3,74 \pm 0,14$ t/ha et $8,57 \pm 0,38$ t/ha sur le site de Lélé. Par contre, avec les engrais perlés, la variété NERICA-L14 a présenté respectivement les rendements grain de riz paddy et paille suivants : $4,33 \pm 0,37$ t/ha et $8,11 \pm 0,57$ tha sur le site de Koussin et $3,54 \pm 0,36$ t/ha et $6,93 \pm 0,83$ t/ha sur le site de Lélé. En général, considérant l'ensemble des traitements d'engrais, on observe une augmentation significative de 1,3 fois du rendement paille avec la variété NERICA-L14 comparativement à celui de la variété IR841 sur le site de Koussin. A l'opposé, la variété IR841 a été plus productive en ce qui concerne le rendement grain de riz paddy comparativement à la variété NERICA-L14. Toutefois, cette différence n'est pas significative. Sur le site de Lélé, c'est la tendance inverse qui s'observe. La variété IR841 a été plus productive que le NERICAL14. On observe une augmentation significative de 1,12 fois du rendement grain de riz paddy avec la variété IR841 comparativement à celui de la variété NERICA-L14. Par contre, cette différence est beaucoup plus prononcée avec le rendement paille. Elle est de 1,2 fois plus importante comparativement au rendement paille de la variété NERICA-L14. Enfin, les résultats montrent également que, le rendement grain de riz paddy est significativement plus élevé au niveau du site de Koussin comparativement au résultat de Lélé. En effet, il est 1,2 fois plus important au niveau de Koussin comparativement au site de Lélé. 

des variétés de riz IR841 et NERICA-L14 au Sud-Bénin

\section{DISCUSSION}

Influence des formes d'engrais minéraux sur les paramètres de croissance du riz: Les combinaisons d'engrais SG-Urée + SG-NPK ainsi que SG-Urée + NPK perlé ont induit des hauteurs des plants, de production de talles et de grains par panicule significativement plus élevées comparativement aux autres combinaisons d'engrais quel que soit la variété de riz et le site de l'essai. Ces résultats témoignent de la nette importance de l'apport de l'urée sous la forme super granulée à la culture de riz de bas-fond en raison de la présence permanente de l'eau dans le milieu. L'effet négatif induit par les apports d'engrais minéraux contenant de l'urée perlée s'expliquerait par l'indisponibilité de l'azote dans le sol qui se serait probablement volatilisé sous la forme $\mathrm{NH}_{3}$ juste après l'application. Selon la FAO (2011), la quantité d'azote disponible dans le sol est prélevée par la végétation ou perdue soit par lessivage ou soit par volatilisation. $\mathrm{Ce}$ résultat est en partie imputable à un effet dépressif de l'engrais (urée et NPK perlés) sur la croissance des plants de riz. Ces effets négatifs des engrais perlés peuvent également relever du mode d'épandage à la volée lequel contribue fortement à la perte des nutriments dans le cas de l'azote en milieu humide (Saïdou et Kossou, 2009). L'idéal étant de distribuer le plus uniformément possible l'engrais aux plants après avoir effectué un drainage de l'eau des casiers rizicoles afin d'éviter les pertes. Dans ce contexte d'une application à la volée des engrais minéraux sous la forme de fumure de couverture, le gaspillage est plausible, car les engrais tombés entre les lignes et entre les pieds, profiteraient plus aux adventices qu'à la culture principale qui subirait une carence accrue. De plus, l'épandage à la volée engendre de faible recouvrement de l'azote (Bandaogo, 2010). Selon le même auteur, ces méthodes sont inefficaces sur le plan agronomique et économique et nuisent à l'environnement. Les performances agronomiques induites par les formes super granulées des engrais témoignent de l'adéquation de ces apports avec les conditions de bon développement des plants de riz ; ce qui explique le grand nombre de talles et de grains par panicule. De plus, ces combinaisons ont graduellement libéré les nutriments en particulier l'azote qui assure l'évolution des différentes phases de croissance et développement de la plante, conférant ainsi, les résultats meilleurs observés au niveau des deux sites avec les deux variétés de riz cultivées. Ce résultat corrobore ceux de Sen et Pandey (1990) qui ont trouvé que l'azote doit être mis à la disposition de la plante à une vitesse qui est égale à celle avec laquelle la plante absorbe cet élément. Bien de travaux ont montré que le placement profond de l'urée super granulée comporte de nombreux avantages agronomiques et socioéconomiques (Pasandaran et al., 1999 ; Mohanty et al., 1999 ; IFDC, 2003 ; Segda et al., 2006 ; IFDC, 2009 ; Traoré, 2009; Yameogo et al., 2013). Les recherches de l'IFDC (2011) et Yameogo et al. (2013) ont prouvé que l'efficience de l'azote peut être nettement améliorée à travers le placement profond de l'urée. En effet, cette technologie entraine une utilisation efficiente de l'azote en le gardant plus dans le sol, hors de l'eau de surface où il est susceptible de se perdre sous forme gazeuse ou par percolation ou encore par écoulement (Mohanty et al., 1999; Traoré, 2009; Yameogo, 2013). Ainsi, les combinaisons d'engrais à base de super granules (SG-Urée + SG-NPK) d'une part, et SG-Urée + NPK perlé d'autre part, ont assuré une remarquable nutrition azotée et phosphatée de la plante, ce qui s'est également traduit par l'importante production de grains observée. Ces combinaisons d'engrais ont considérablement induit un bon développement des plants de riz. On pourrait recommander ces formes d'engrais pour accroître la production du nombre de talles du riz élément précurseur du rendement grain et paille. Le faite que l'engrais NPK soit appliqué sous la forme super granulée a contribué à une amélioration de l'efficience du phosphore traduit par une importante production de grains par panicule au regard des fonctions physiologiques de ce nutriment (Elalaoui, 2007).

Influence de l'application des différentes formes d'engrais minéraux sur les rendements grains de riz paddy et paille: II ressort des résultats de la présente étude que, l'application des engrais super granulés ont beaucoup plus induit une augmentation des rendements grains de riz paddy et paille au niveau des deux variétés comparativement à l'application des engrais perlés. La variété IR841 a donné des rendements riz paddy sur le site de Koussin similaires aux résultats des travaux de Traoré (2009) au Burkina Faso après utilisation d'urée super granulée. Toutefois, les résultats obtenus avec la variété NERICA-L14 sur les deux sites sont légèrement inférieurs à ceux obtenus par l'auteur (entre 4,73 et 5,77 t/ha contre 6,03 t/ha au Burkina Faso) bien qu'ils restent similaires a ceux obtenus au Sud de la Côte d'Ivoire sous l'effet de différents types de fertilisants (Kouakou et al., 2014). Ceci dénote l'effet écologique sur la performance et l'efficacité de la variété NERICA-L14 à mieux valoriser 

des variétés de riz IR841 et NERICA-L14 au Sud-Bénin

les nutriments fertilisation chimique. Les faibles résultats obtenus avec les engrais appliquées sous la forme perlée comparativement à l'application sous la forme super granulée s'expliquent par la non valorisation de l'azote qui se serait probablement volatilisé dans cet environnement humide avec présence permanente d'eau. En effet, les pertes en azote par volatilisation et par nitrification/dénitrification sont réduites avec l'utilisation des engrais azotés super granulés (Mohanty et al., 1999; Pasandaran et al., 1999 ; Segda et al., 2006 ; Yameogo et al., 2013). De plus, l'urée super granulée est apportée à une surface très réduite du sol. Ainsi, lors de l'hydrolyse, les ions ammoniums se fixent progressivement sur les colloïdes du sol, ce qui réduit les pertes par volatilisation. Or, avec l'urée perlée, les pertes sont souvent très énormes et peuvent atteindre 60 à $70 \%$ (Pasandaran et al., 1999 ; IFDC, 2011). A ces pertes énormes s'ajoute le manque de synchronisation entre l'offre et la demande en nutriments surtout l'azote (Segda et al., 2004 ; 2005) en ce sens que, l'urée simple après hydrolyse, diffuse rapidement en 3 à 4 jours. Cet azote libéré ne peut donc pas satisfaire tous les besoins de la plante qui sont étalés dans le temps (Elalaoui, 2007) raison pour laquelle il est recommandé de procéder à un fractionnement de l'apport pour les céréales. L'importance de l'utilisation du super granule d'urée dans l'amélioration de la nutrition minérale et la productivité du riz s'est fait alors ressentir avec un accroissement du gain de rendement tel que observé dans la présente étude. Ces résultats obtenus sont en conformité avec ceux obtenus par Bandaogo (2010) pour lesquels l'urée super granulée a été plus productive que l'urée ordinaire dont le gain en rendement était supérieur de 1 tha. Selon la FAO (2011), l'utilisation de l'urée super granulée provoque une augmentation du rendement paddy de 15 à $25 \%$ comparativement à l'urée perlée. Cette différence de rendements a été également obtenue dans la présente étude et observée dans de nombreux pays d'Afrique où

\section{CONCLUSION}

L'application des différentes formes d'engrais minéraux en riziculture de bas-fond au sud Bénin donne une meilleure compréhension de l'importance de la fertilisation raisonnée. Les combinaisons urée et NPK super granulés et urée super granulée et NPK perlé ont toute induit de meilleurs performances agronomiques (croissance en hauteur, production de talles et grains par panicules, rendement paddy et paille) indépendamment des variétés de riz et des sites les performances de l'urée super granulée ont été toujours nettement supérieures à celle de l'urée perlée (Chowdhury et al., 2009 ; Bandaogo, 2010 ; Yameogo, 2013). Des observations similaires ont été faites sur d'autres cultures comme le chou où les rendements induits par l'urée super granulée sont nettement supérieurs à ceux de l'urée perlée (Hussain et al., 2010). Des travaux réalisés par l'IFDC (2003) sur l'utilisation de l'urée super granulée il ressort que, cette technologie entraîne une augmentation du rendement de 1,2 t/ha de paddy en moyenne. Ces différentes observations corroborent nos résultats au niveau des deux sites avec les deux variétés cultivées. En général, il ressort de nos résultats que la combinaison SG-Urée + NPK perlé a été plus performante en terme de rendement grains riz paddy et paille comparativement à la combinaison urée perlée + SGNPK. Cela est dû au fait que, les pertes en azote au niveau de l'urée perlée sont plus importante que celles observées au niveau du SG-NPK. Cela est perçu au niveau de tous les graphes présentés et qui se matérialise par des différences non significatives entre la combinaison urée perlée + SG-NPK et la combinaison urée et NPK perlés. La principale conclusion qui ressort de la présente étude est qu'un accent particulier doit être mis sur la granulation de l'urée dans le cadre de la formulation des engrais pour la production du riz de bas-fonds en général. A cet effet, les producteurs de riz du Bénin en zone de basfond gagneraient sur le plan financier en utilisant l'urée et le NPK sous la forme super granulée. Cette technologie permettrait de réduire les quantités d'engrais actuellement utilisées dans la zone et d'accroitre l'efficience de l'utilisation de l'azote et le niveau de rendement de la culture. Par conséquent, la pratique permettrait d'améliorer le niveau de revenu obtenu de la production du riz puis réduira le niveau de pollution de l'eau due à l'utilisation des engrais azotés dans les zones humides.

comparativement aux engrais perlés. II est apparu de nos résultats qu'une meilleure valorisation des nutriments des engrais super granulés était intrinsèquement liée aux caractéristiques de la variété de riz cultivée. Ainsi, sur les deux sites, la variété IR841 a été beaucoup plus performante pour les paramètres mesurés comparativement à la variété NERICA-L14. De plus, les pratiques culturales des producteurs doivent être prises en compte afin d'améliorer 
l'efficience de l'utilisation des nutriments par la plante explication plausible des écarts de rendement observés entre les sites de Koussin et celui de Lélé. La technologie des engrais super granulés en riziculture irriguée contribuera surement à une amélioration de la production locale du riz au Bénin.

\section{REMERCIEMENTS}

Les auteurs remercient l'IFDC Afrique Lomé Togo pour avoir financé les activités de recherche ayant aboutit à la rédaction du présent article.

\section{REFERENCES BIBLIOGRAPHIQUES}

Abiassi E., 2006. Etudes sur les instruments de régulation des importations commerciales de riz au Bénin. Rapport CCR-FUPRO. Bohicon, Bénin, $80 \mathrm{p}$.

Adégbola Y.P. et Sodjinou E. 2003. Etude de la compétitivité de la riziculture béninoise. Rapport définitif, PAPAIINRAB et ADRAO. Cotonou Bénin, $58 \mathrm{p}$.

ADRAO, 2007. Tendances rizicoles en Afrique : Vue d'ensemble sur l'évolution du secteur rizicole en Afrique Subsaharienne. Synthèse $d u$ Centre du riz pour l'Afrique. ADRAO. Cotonou, Bénin, $10 p$.

Agbobli C., Adomefa K., Labare K., 2007. Situation de référence sur les principales céréales cultivées au Togo : Maïs-Riz-Sorgho-Mil. ITRA, Lomé Togo, $107 \mathrm{p}$.

Bandaogo A.A., 2010. Effet de différentes sources d'azote sur la réponse de quatre variétés de riz à l'azote dans la Vallée du Kou au Burkina Faso. Mémoire du Diplôme d'Etudes Approfondies (DEA), Institut du Développement Rural, Université Polytechnique de Bobo-Dioulasso, BurkinaFaso, $45 \mathrm{p}$.

Chowdhury A.K.M.S.H., Kabir M.H., Sarkar M.A.R., 2009. Effect of urea super granules, prilled urea and poultry manure on the yield of transplant Aman rice varieties. Bangladesh Agricultural University, 7(2) : 259-263.

CPCS-Commission de pédologie et de cartographie des sols, 1967. Classification des sols. Tableaux des classes, sous-classes, groupes et sous-groupes des sols. Service de classification des sols. INRA, France. 96 pp.

Elalaoui C.A., 2007. Fertilisation minérale des cultures: les éléments fertilisants majeurs (azote, phosphore, potassium). Bulletin mensuel d'information et de liaison du PNTTA, Transfert de Technologie en Agriculture $N^{\circ} 155$. Ministère de l'Agriculture et de la Pêche Maritime, Royaume du Maroc. 4 p.
Fagade, S.O., 2000. Yield gaps and productivity decline in rice production in Nigeria. In: FAO (Ed.), Proceedings of the Expert Consultation on Yield Gap and Productivity Decline in Rice, Rome, Italy. pp. 15-37.

FAO, 2004. Le riz c'est la vie. Bulletin trimestriel de statistiques. Vingt troisième conférences régionales pour l'Afrique, année internationale du riz. 1-5 mars 2004, Johannesburg. FAO, Rome Italie.

FAO, 2011. Produire plus avec moins d'intrant. Guide à l'intention des décideurs sur l'intensification durable de l'agriculture paysanne. FAO, Rome, Italie.

FAOSTAT, 2012. Food and Agriculture of the United Nations Database. FAO, Rome Italy.

Hussain M.J., Alim Y., Rahman M.A., Quayyum A., Choudury D.A., 2010. Effect of urea super granule on the performance of cabbage in young Jamuna and Brahmaputra floodplain soils of Tangail. Bangladesh Journal of Agricultural Research, 35(2) : 267-272.

IFDC, 2003. More rice with reduced loss of urea. An IFDC project to improve fertilizer nitrogen efficiency for rice production. IFDC, Lomé Togo. $11 \mathrm{p}$.

IFDC, 2009. An uptake on the work and progress at IFDC. Vol 34, $\mathrm{N}^{\circ} 3,12 \mathrm{p}$.

IFDC, 2011. La technologie du Placement Profond de I'Urée peut contribuer de manière significative à la réalisation de l'autosuffisance en riz en Afrique de l'Ouest. Projet MIR Plus et I'Initiative PPU/SAADA. Fiche d'information, $n^{\circ} 6.4 p$

IFDC, 2012. Gestion intégrée du riz irrigué. Fiche technique $\mathrm{N}^{\circ} 10$. Kigali-Rawanda, Février 2012. IFDC, Lomé Togo.

Koné B., Amadji L.G., Saïdou A., Diatta S., Akakpo C., 2011. Nutrient constraint and yield potential of rice on upland soil in the south of the Dahomey gap of West Africa. Archives of Agronomy and Soil Science, 57(7): 763-774. 
Kouakou O., Mamadou C., Koné B., Dick E., Konan K. F., 2014. Growth, yields and rooting performance of lowland rice NERICA L14 as affected by different fertilizers. Ind. J. Sci. Res. and Tech. 2014 2(2):18-24.

MAEP, 2010. Rapport d'analyse de l'insécurité alimentaire et des inégalités d'accès à l'alimentation au Bénin. Cotonou, Ministère de l'Agriculture de l'Elevage et de la Pêche, Cotonou Bénin, $27 \mathrm{p}$.

Mohanty S.K., Singh U., Balasubramanian V., Jha K.R., 1999. Nitrogen deep-placement technologies for productivity, profitability and environmental quality of rainfed lowland rice systems. Nutrient Cycling in Agroecosystem, 53 : 4347.

PAGFS/MAEP, 2010. Actualisation de la carte de fertilité des sols du Bénin. Rapport provisoire amélioré : par zone agro-écologique. Programme d'Appui à la Gestion de la Fertilité des Sols (PAGFS)/Ministère de l'Agriculture de l'Elevage et de la Pêche (MAEP), Cotonou, Bénin.

Pasandaran E., Gulton B., Adiringsih J.S.R.I., Aspari H., Rochayati S.R., 1999. Government policy support for technology promotion and adoption: a case study of urea tablet technology in Indonesia. Nutrient cycling in Agroecosystems, 53 : 113- 119.

Ponnamperuma, F. N., 1972. The chemistry of submerged soils. Advance Agronomy 24, 29 96.

Roger P.A., 1993. Les bio fertilisants fixateurs d'azote en riziculture: potentialités, facteurs limitant et

perspectives d'utilisation. In Raunet M. (ed.) Bas-fond et riziculture. CIRAD, Montpellier, France. pp 327-348.

Saïdou A. et Kossou D., 2009. Water management for enhancing land productivity in Benin: perceived constraints and opportunities for the development of smallholder farmers. In: A. van Huis and A. Youdeowei (eds). Proceedings of the 1st CoS-SIS International Conference, 2226 June, Elmina, Ghana, pp. 48-52.

Segda Z., Haefele S.M., Wopereis M.C.S., Sedogo M.P., Guindo S., 2004. Agroeconomic characterization of rice production in a typical irrigation scheme in Burkina Faso. Agronomy Journal, 96 : 1313-1322.

Segda Z., Haefele S.M., Wopereis M.C.S., Sedogo M.P. and Guindo S., 2005. Combining field and simulation studies to improve fertilizer recommendations for irrigated rice in Burkina Faso. Agronomy Journal, 97 : 1429-1437.

Segda Z., Sie M., Mando A., Haefele M.S., Wopereis M.C.S., Kebbeh M., Miezan K., Sedogo M.P., Guindo S., 2006. Performances d'une gestion intégrée des nutriments pour la production de riz irrigué dans la plaine de Bagré au Burkina Faso. Agronomie Africaine, $\mathrm{N}{ }^{\circ}$ Spécial 5 , année internationale du riz, 29-39.

Sen A. and Pandey B.K., 1990. Effect of placement depth of urea super granules on rice. International Rice Research News, 15: 51.

Totin E., van Mierlo B., Saïdou A., Mongbo R., Agbossou E., Stroosnijder L., Leeuwis C., 2012. Barriers and opportunities for innovation in rice production in the inland valleys of Bénin. NJAS - Wageningen Journal of Life Sciences, 60-63: 57-66.

Totin E., Stroosnijder L., Agbossou E., 2013. Mulching upland rice for efficient water management: A collaborative approach in Benin. Agricultural Water Management, 125 : 71-80.

Traoré M., 2009. Contribution de l'urée super granulée dans l'amélioration de la production du riz irrigué sur la plaine de Bagré au Burkina Faso. Mémoire d'Ingénieur de Développement rural. Université de Bobo Dioulasso, Burkina Faso. $64 \mathrm{p}$.

Yameogo L., Zegda Z., Dakouo D., Sedogo M.P., 2013. Placement profond de l'Urée (PPU) et amélioration de l'efficacité d'utilisation de l'azote en riziculture dans le périmètre rizicole de karfiguela au Burkina Faso. Journal of Applied Biosciences 70 : 5523 - 5530. 